\title{
Enfermedad de Ledderhose familiar
}

\author{
Eva Buller Viqueira ${ }^{a}$, Esperanza Buller Viqueirab ${ }^{b}$ Juana Cabello Pulido ${ }^{a}$
}

a Centro de Salud Gonzalo Pérez Fabra. Paterna de Rivera. Cádiz (España).

b Asistencial Europa Reifs. Chiclana. Cádiz (España).

Correspondencia: Eva Buller Viqueira. Centro de Salud Gonzalo Pérez Fabra. C/ Molino, 14. 11178 - Paterna de Rivera. Cádiz.

Correo electrónico: miji_77@yahoo.com

Recibido el 28 de agosto de 2013.

Aceptado para su publicación el 16 de septiembre de 2013.

\section{RESUMEN}

La enfermedad de Ledderhose es una hiperproliferación benigna de fibroblastos y colágeno a nivel de la aponeurosis plantar. Patología de baja incidencia y considerada por algunos autores como enfermedad rara. Más inusual es la presentación familiar.

Presentamos el caso de una mujer de 63 años con enfermedad de Ledderhose desde la adolescencia además de enfermedad de Dupuytren (enfermedades que pueden verse vinculadas con frecuencia). Familia de 9 hermanos con 4 afectados de fibromatosis plantar y 6 con enfermedad de Dupuytren. Otros familiares también afectados.

Esta enfermedad no es muy conocida en Atención Primaria, probablemente por su baja incidencia. Vemos conveniente repasar la patología para su correcto tratamiento y derivación cuando precisara. Además sería interesante la localización de familiares afectos para estudio genético.

Palabras clave: Fibromatosis Plantar Familiar. Enfermedad de Ledderhose. Herencia. Enfermedades del pie.

\section{ABSTRACT}

\section{Family Ledderhose disease.}

Ledderhose disease is a benign hyperprofilation of fibroblasts and collagen at plantar aponeurosis level. A low incidence pathology and considered by some authors as a rare disease. Whole family involvement is even more unusual. We present the case of a sixty-three year old woman suffering from Ledderhose disease from adolescence, along with Dupuytren's disease (diseases which can frequently be linked). A family of nine siblings, four of whom being affected by plantar fibromatosis, and six with Dupuytren's disease. Other family members also affected. This disease is not very well known in primary care, probably due to its low incidence. We believe it is recommendable to review the pathology for its correct treatment and referral where necessary. It would also be of interest to locate affected family members for genetic study. Key words: Family plantar fibromatosis. Ledderhose disease. Heredity. Foot Diseases.

\section{INTRODUCCIÓN}

La enfermedad de Ledderhose (EL) o fibrosis plantar fue descrita por primera vez en 1897 por el cirujano Georg Ledderhose. Se caracteriza por una hiperproliferación benigna de fibroblastos y colágeno a nivel de la aponeurosis plantar, de crecimiento lento. Es el equivalente a la enfermedad de Dupuytren (ED), pero a nivel plantar'1.

La incidencia de la ED está bien definida, en cambio la de la fibrosis plantar no es tan conocida. Fue descrita y registrada en EE.UU. como enfermedad rara, presentando una incidencia de menos de 200.000 casos en la población de EE.UU. Es diez veces más predominante en el sexo masculino y presenta mayor afectación en la raza blanca. Suele aparecer en la edad media de la vida y asociarse a enfermedades como la diabetes, la epilepsia, enfermedades hepáticas crónicas, etilismo crónico, otras fibromatosis y traumatismos ${ }^{2}$. El $50 \%$ de los casos de enfermedad de Ledderhose padecen además ED, pero inversamente la fibrosis palmar se acompaña de la plantar sólo en el 3\% de los casos. Se ha descrito una herencia autosómica dominante en la ED, pero son raros los casos de fibromatosis plantar familiar. La coexistencia 
de la fibromatosis de la aponeurosis plantar con la fibromatosis de los cuerpos cavernosos o enfermedad de Peyronie es rara ${ }^{2,3}$.

\section{OBSERVACIONES CLÍNICAS}

Presentamos el caso de una mujer de 63 años con diagnóstico de EL. Acude a nuestra consulta por sensación de cuerpo extraño en el zapato del pie izquierdo, que describe como "si tuviera una piedra". No se acompaña de dolor, aunque si refiere "molestia" a la palpación de la región afectada. Esto corresponde a una recidiva de su enfermedad. No se observa la presencia de retracción de los dedos del pie afectado (figura 1). Durante la entrevista manifiesta su interés por someterse a una nueva intervención quirúrgica. Los primeros síntomas de su patología aparecieron a los 15 años de edad. Fue intervenida por primera vez en el año 1981, precisando una nueva intervención en 1993. Aproximadamente a los 50 años de edad fue diagnosticada de ED de afectación bilateral.

Importante historia familiar tanto de ED como de EL. No existen datos médicos, pero sí conocimiento

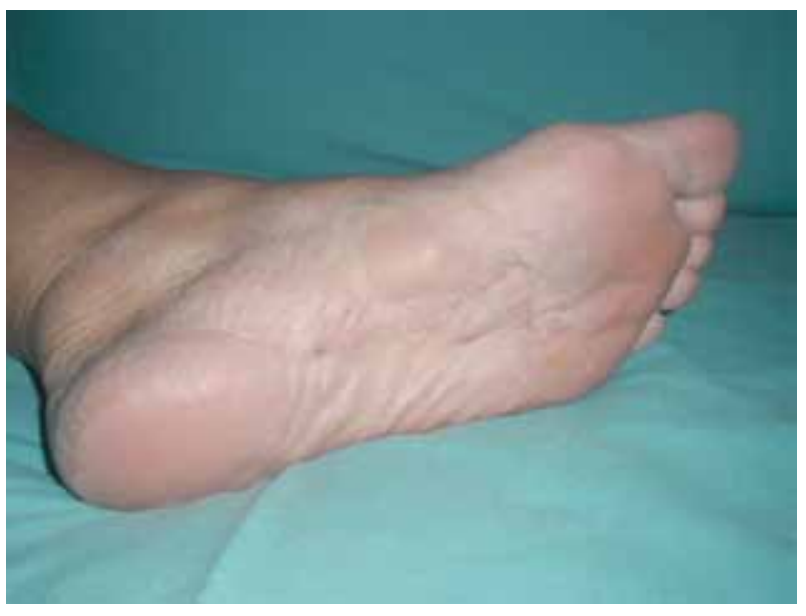

Figura 1. Enfermedad de Ledderhose en el caso presentado por parte de los familiares de que la abuela paterna y su padre tuvieran ED, sin saber en ninguno de los dos casos si podría haber también EL. Sí hay diagnóstico médico en todos los demás casos descritos a continuación. Familia de 9 hermanos (figura 2):

- $\quad 1^{\circ}$ Mujer (el caso) EL desde los 15 años en pie izquierdo, 2 intervenciones quirúrgicas, nuevamente recidiva. ED bilateral desde los 50 años. Tiene cuatro hijas de las que una padece EL desde los 3 años de edad, intervenida en 2 ocasiones y nuevamente recidiva.

- $2^{\circ}$ Mujer no afectada.

- $3^{\circ}$ Varón (Mellizo) ED bilateral.

- $4^{\circ}$ Varón (Mellizo) EL bilateral desde los 30 años aproximadamente y ED bilateral desde los 43 años.

- $5^{\circ}$ Mujer ED.

- $6^{\circ}$ Mujer no afectada.

- $7^{\circ}$ Mujer no afectada.

- $\quad 8^{\circ}$ Varón con EL desde los 12-13 años de edad intervenido y recidiva en 2 ocasiones. ED bilateral desde los 43 años.

- $\quad 9^{\circ}$ Varón EL bilateral a los 22 y 25 años de edad.

Además, un tío abuelo paterno del caso tuvo sospecha de ED, su hijo tiene diagnóstico médico y la hija de éste también.

\section{COMENTARIOS}

Dado que la EL es una patología poco frecuente y desconocida por muchos médicos de Atención Primaria, vemos conveniente repasar esta patología.

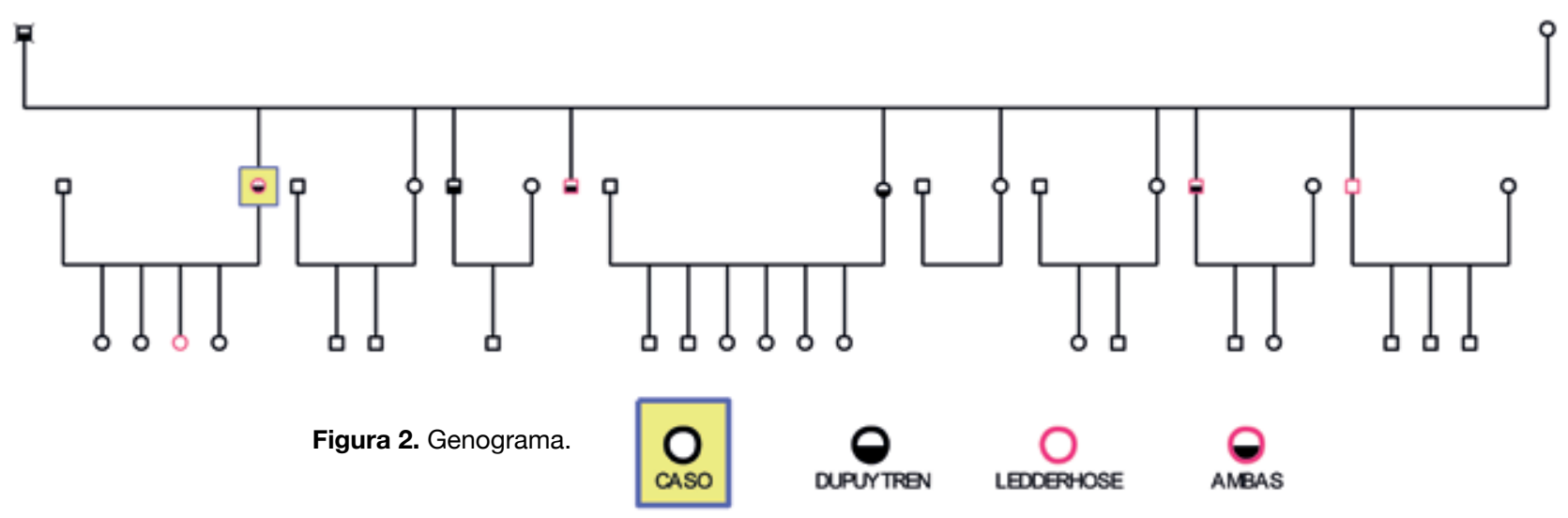


La EL se puede presentar como un único nódulo fusiforme o fibroma, como varios fibromas o como una masa infiltrante (fibromatosis). Su localización más común es la parte central y medial de la fascia plantar. Las lesiones más superficiales son de menor tamaño, mejor definidas y menos agresivas que las profundas. Estas últimas pueden afectar al músculo, estar peor definidas y no detectarse por su profundidad hasta hacer efecto masa y provocar dolor. Puede ser asintomático en un comienzo y después apreciarse una sensación de cuerpo extraño en el zapato, dificultad para calzarse y, a medida que aumenta de tamaño, dolor a la bipedestación y a la marcha. El tamaño es variable. A la exploración los nódulos se suelen deslizar bajo la piel, tras una larga evolución terminan por adherirse. Al tensionar la aponeurosis con la extensión de los dedos del pie, permite apreciar mejor el nódulo. No se acompaña de signos inflamatorios, la piel subyacente es normal y no hay cambios de temperatura ${ }^{4}$.

La EL y la ED son manifestaciones de hiperplasia de tejido fibroso que presentan una gran analogía, pero con ciertas diferencias. Existe la teoría de que son dos expresiones de la misma enfermedad ${ }^{5}$. En la EL las lesiones son a nivel del pie en lugar de la mano, los nódulos suelen alcanzar un tamaño mayor, rara vez produce retracción de los dedos por la propia anatomía de la fascia plantar, no suele haber un engrosamiento del cordón tan evidente. La terapia en la fibrosis plantar va más encaminada a disminuir el tamaño de los nódulo para aminorar el dolor, la inflamación y mantener una correcta deambulación. En la ED se trata la retracción de los dedos (tabla 1).

El diagnóstico de elección es la resonancia nuclear magnética que delinea con mayor precisión la extensión de la lesión, pero siempre acompañada con una clínica compatible ${ }^{4}$.

El tratamiento puede ser médico (fisioterapia, ortoprótesis, AINEs, infiltraciones de esteroides y anestesia para disminuir el dolor) o quirúrgico (fasciectomía parcial o total con o sin radioterapia postoperatoria para disminuir las recidivas). La cirugía se indica cuando la masa es dolorosa y no se ha podido controlar con tratamiento médico, importante tamaño, dificultad para calzarse 0 aumento en la velocidad de crecimiento. Hay altas incidencias de recidivas tras el tratamiento quirúrgico ${ }^{6}$.

La EL tiene una muy baja incidencia y es considerada por algunos autores como enfermedad rara, motivo por el cual no es una patología muy conocida en Atención Primaria. Son escasos los casos de fibromatosis plantar familiar y por ello dignos de destacar. Creemos que sería recomendable familiarizarse con esta patología para su correcta derivación y para valorar posibles casos entre familiares, muy importante labor en Atención Pimaria. Además, sería interesante aprovechar casos familiares con varios individuos afectos como éste para ampliar conocimientos referentes a la transmisión genética.

\section{BIBLIOGRAFÍA}

1. Lee TH, Wapner KL, Hecht PJ. Plantar fibromatosis. J Bone Joint Surg Am. 1993; 75 (7): 1080-4.

2. Fausto de Souza D, Micaelo L, Cuzzi T, Ramos-e-Silva M. Ledderhose disease: an unusual presentation. J Clin Aesthet Dermatol 2010; 3 (9): 45-7.

3. Graells Estrada J, García Fernández D, Badia Torroella F, Moreno Carazo A. Familial plantar fibromatosis. Clin Exp Dermatol. 2003; 28 (6): 666-70.

4. Chokshi FH, Jose J, Clifford PD. Plantar fibromatosis. Am J Orthop 2009; 38 (9): 475-9.

5. Banerjee S, Muhammad M, Chinmoy N, Kumar D. Plantar fibromatosis: a case report. Foot Ankle Online J. 2009; 2 (12): 3.

6. Sammarco GJ, Mangone MG. Classification and treatment of plantar fibromatosis. Foot Ankle Int. 2000; 21 (7): 563-9.

\begin{tabular}{lcc}
\hline & Ledderhose & Dupuytren \\
\hline Localización & Cara medial del arco plantar & Cara palmar del $4^{\circ}$ ó $5^{\circ}$ dedo \\
Consistencia & Nódulo gomoso, no adherido a piel, bien definido & Nódulo duro, engrosamiento en cordón \\
Descripción más frecuente & Sensación de cuerpo extraño en el zapato & Tirantez \\
Piel & Sin cambios & Engrosada \\
Retracción & No es frecuente & Frecuente \\
\hline
\end{tabular}

Tabla 1: Manifestaciones clínicas más comunes de la enfermedad de Ledderhose y Dupuytren 